\title{
LE-OLSR protocol performance evaluation in various energy conditionsof mobile ad-hoc and sensor wireless networks
}

\author{
M. Belkheir ${ }^{1}$, M. Rouissat ${ }^{2}$, A. Mokaddem ${ }^{3}$, M. Bouziani ${ }^{4}$, A. Zerroug ${ }^{5}$ \\ ${ }^{1,3}$ Instrumentation and Advaned Materials Laboratory, NourBachir University Center, Algeria \\ ${ }^{2}$ STIC Laboratory of Tlemcen, University AboubekBelkaid, Algeria \\ ${ }^{4,5}$ Laboratory of Telecommunications and Digital signal Processing, University of Djillali Liabes, Algeria
}

\begin{tabular}{l} 
Article Info \\
\hline Article history: \\
Received Oct 9, 2019 \\
Revised Mar 24, 2020 \\
Accepted Apr 20, 2020 \\
\hline
\end{tabular}

\section{Keywords:}

Energy optimization

LE-OLSR

Mobile ad hoc networks Network lifetime QoS

\begin{abstract}
A mobile Ad hoc and sensor wireless network commonly includes various mobile devices and sensors with limited resources. In such network, energy constraint and link stability are the most critical parameters to take into consideration when deploying routing mechanisms, to assure reliable communication between nodes. These requirements are due to the network scalability and the absence of a fixed infrastructure, which may cause a rapid depletion of the nodes batteries and leads onto network link breakages. LE-OLSR (Lifetime Enhancement - Optimized Link State Protocol) is a variant of the existing OLSR. This latter is widely used as routing protocol for mobile ad-hoc and wireless sensor networks. LE-OLSR implements a new load balancing algorithm; it takes into account energy constraints of a node when selecting routers, responsible for forwarding data packets over the entire network. The aim of our work is to study and simulate the LE-OLSR protocol behavior in realistic mobile environments, including scenarios with various residual energy constraints schemes. The performed simulations had shown that LE-OLSR performances decrease compared to the case of ideal mobile network. Nevertheless, LE-OLSR had shown persistence and fair results in the realistic environment. The obtained results show that LE-OLSR protocol is an adequate and a prime solution for dense mobile ad-hoc networks.
\end{abstract}

Copyright $\odot 2020$ Institute of Advanced Engineering and Science. All rights reserved.

\section{Corresponding Author:}

Mohammed Belkheir,

Instrumentation and Advaned Materials Laboratory,

Nour Bachir University Center,

32000, El-Bayadh, Algeria.

Email: belkheir_m@yahoo.fr

\section{INTRODUCTION}

Energy remains one of the critical resources in mobile ad hoc networks [1], knowing that nodes are battery-powered, with a limited capacity. Afterwards, when the energy deplete in some, it causes subsequently a loss of connectivity and leads to a network partitioning. Hence, for effective deployment of large scale ad hoc networks, it is necessary to develop mechanisms that take into account the limited battery life of mobile devices.

Moreover, the absence of a centralized administration and a fixed infrastructure make the implication of all network nodes mandatory, especially in the packet forwarding process. Within mobile ad hoc networks the connectivity is ensured by each node, which is required to play the role of host and router to guarantee the traffic delivery activity [2]. Hence, any node can be sought to contribute in various operations, like: path discovery, routing packets and also for the route maintenance process when the topology change. This permanent activity of nodes within a mobile ad hoc networks draws of their limited resources, where each node should keep listening to the channel in order to intercept and rebroadcast control packets. 
Moreover, the free mobility of nodes requires challenges for routing data in such networks [3]. Route failures produce a permanent appeal of the discovery and the maintenance process implementing by routing protocols [4]. Therefore, a new routing mechanism should keep energy optimization as a most criterion in order to assure network services for a long time as possible [5]. The link breakage between nodes may be caused by many reasons, such as a failure of an intermediate node or a discharge of its battery [6]. This situation is so critical for such networks and creates holes which make some nodes unreachable. To avoid this concern, routing protocols should be energy aware, and routes established between nodes should be maintained by electing nodes with a high energy level [7].

In the literature, a variety of routing protocols designed for mobile ad hoc and sensor wireless network have been proposed, taking into account the energy constraints of a network, in order to enhance the efficiency in terms of a reliable data transfer and to assure a desired level of Quality of Service (QoS). [8, 9]

OLSR (Optimized Link State Routing) [10], is one of proactive protocols, widely used in mobile ad hoc and sensor wireless networks. The optimization process in OLSR is related to multipoint relay (MPR) concept. An MPR is a node which is selected as a router among its neighbors due to its position. The MPR will play a role of a router responsible of forwarding data packets coming from its neighbors. MPRs are also responsible for forwarding control and topology packets for route discovery process. This aims to minimizethe flooding of control traffic. OLSR contains two types of control messages: neighborhood and topology messages, known as Hello messages and Topology Control (TC) messages. OLSR provides two main features: Neighbor Discovery and Topology Dissemination. With the help of these two main features, each node will be able to construct a set of routes over the entire network [10-13].

Moreover, the established routes may not be energy efficient in OLSR. To enhance the QoS of mobile ad hoc networks using OLSR as routing protocol, the MPRset selection process should include the residual energy level as a criterion, in addition to the position of a node within the network. The association of those two parameters allow a node to elect a set of nodes among its neighbors as MPRs that can be energy aware and may to relieve nodes which has been selected as routers for a long time, due to their position. That is why, in a previous work, we updated the heuristic of the basic OLSR for performing the MPR set, to take into account both energy level of a node and its reachability degree. We introduced a mechanism to enhance the creativeness of a node in order to give it more chance to become a router. By this way, we assure a load-balancing for routing activityupon the entire network. Anew variant of OLSR was named LE-OLSR (Lifetime Enhancement-OLSR) [14], which represents the basis of our work and will be detailed in the following sections of our present paper. A second variant named WRE-OLSR (Weighting between reachability and energy - OLSR) [15], that implements a new process for the MPRset selection, based on a weighting between the position of a node in the network and its residual energy. Despite the highest reachability level of a node due its position, it may not be selected as MPR if its energy becomes low. Hence, we can avoid holes in the network core, caused by the permanent solicitations of nodes as routers due to their position. Simulation results in $[14,15]$ have shown an enhancement in the quality of service (QoS) parameters, like the control overhead, the average end to end delay and dead nodes, by implementing these new protocols. Consequently, this process increases significantly the lifetime of the network, even when the energy resources of nodes becomes low.

In this paper, we focused on studying LE-OLSR [14], performances when it's implemented for a realistic environment, by considering various topologies and energy level scenarios. Realistic ad-hoc networks may be built by nodes with various battery capacities. Thus, we should evaluate and validate our protocol by choosing scenarios which can be close to the reality.

The remaining parts of this paper follow the pattern highlighted as discussed here: the second section presents the protocol LE-OLSR and its contribution to the basic OLSR protocol. The third section includes simulation environment scenarios used in NS-2 simulator. Based on simulations results, in the Section 4, we measure and evaluate the performance of LE-OLSR protocol in a dynamic environment similar to the reality, where the nodes start with a random initial energy, comparing to an ideal scenario where nodes have the same amount of battery capacity. Finally, Section 5 summarizes discussions and concludes this work.

\section{LE-OLSR VS OLSR AND ENERGY OPTIMIZATION SCHEME}

Before discussing about LE-OLSR [14] which is the focal point of our study, let us give a brief description of the OLSR protocol. OLSR is one of the proactive protocols implemented in ad-hoc and sensor wireless networks. This protocol is based on the selection of an MPRset among the node neighbors, which are responsible to relay data from this node to rest of the network, based on the essential criterion which is the reachability of a node (i.e the position of a node) [10]. The RFC (Request For Comment) 3626 defines the first version of OLSR. Afterwards, RFCs 5444 [11] and RFC5497 [12] defined other versions of OLSR, adding some functionalities while keeping the same process of the network core.

Indonesian J Elec Eng \& Comp Sci, Vol. 19, No. 3, September 2020 : 1391 - 1398 
RFC3626 defines the core routing process of OLSR by three different types of messages. Eachnode within the mobile network maintains updated information on the link state by flooding control messages which are "TC" for "Topology Control" and "Hello" in OLSR case. Nodes use the exchanged information to calculate the optimal path to other nodes when desiring to transmit useful data.

OLSR contains a kind of control traffic messages (TC messages), periodically exchanged by nodes to build links map with their neighbors [16]. "Hello" messages are typesof broadcasted messages to discover symmetric linkswith their one-hop neighbors. By this way, nodes may construct their neighbor tables and to select their MPRs. The neighborhood discovery process allows a node to calculate the "MPRset" and another important table named "MPR selector set" that represents nodes which have selected the present node as MPR. After the neighborhood process, the MPRs selected can exchange other control messages called "TC: Topology Control" in order to construct the topology table and declare their selectors.

A previous work in [17], demonstrates that a node playing a role of MPRs for a long-time exhaust rapidly its residual energy, comparing to a non MPR-node. This phenomenon seems very critical and drastically degrade the stability of a mobile ad hoc network, even when MPRs are selected based on their positions. The death of central nodes creates holes and generates link breakages between nodes.

LE-OLSR, uses the "reserved" fields available in HELLO and TC messages to transmit residual energy level information for each node. This information is then associated with the value of the 'willingness' field. The "Willingness" field of a node is specified by an integer varying from 0 to 7 and indicates the ability of a node to play the MPR role. So, nodes should inform their neighbors about their capacities by using the "Willingnes" field in the HELLO packets.

Another aspect has been observed in the functioning of the basic OLSR protocol; when nodes reach their minimum level of capacity (WILL_LOW) they inform other nodes that they are not able to become MPRs. This, decreases the optimum MPR number, increases the control overhead and degrades performances in terms of network lifetime. Control packets are flooded over the entire network and further degrade nodes capacity. To avoid this unwanted situation, LE-OLSR implements the "MPRselset" table which contains nodes which have selected the present node as MPR. This means that if theMPRselset of a node is empty, so the present node has not been selected as MPR due its residual energy or its position in the network. Afterwards, the basic OLSR algorithm of MPR selection is modified by introducing a dynamic threshold, which decreases automatically by one (1) when MPRselset of the node isempty. This process is obviously described by the following algorithm which associates the energy level of a node to its willingness as shown in Figure 1.

Upon receiving a Hello message, a node updates its repositories with information gathered from its neighborhood. An empty MPRselset of the computing node would signify that it has not been selected as MPR due its energy level. Hence, it decreases its willingness level to tell other nodes that it can be MPR for another time in order to avoid the absence of MPRs within the network.

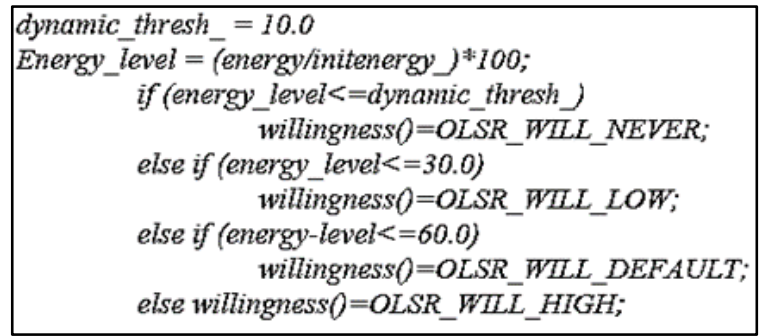

Figure 1. Algorithm (1) for association between the willingness and the energy of a node

\section{RESEARCH METHOD}

Simulations performed in [15] assumed that all nodes of the network have initially the same and the maximum amount of the energy. But, in the reality, amobile ad-hoc network can be randomly built by mobile devices in order to temporarily exchange data. A non-fixed infrastructure of such networks and their mobility intends us to study the efficiency of LE-OLSR within several random scenarios in order to evaluate its QoS performances. In our present research work, we evaluate and analyze the QoS performances of LE-OLSR protocol. The main goal of our analysis is to investigate the behavior of our proposed scheme under two different environments, namely:

a) Static environment with a constant initial energy of 100 joules for each node.

b) Dynamic environment with a variable initial energy within the interval [50 joules, 100 joules].

At first, we performed a script that generates a variable called RNG (Random Number Generation). 
This script allows us to generate random number between 50 and 100. The performed script is written in TCL/tk [18, 19] language as follows:

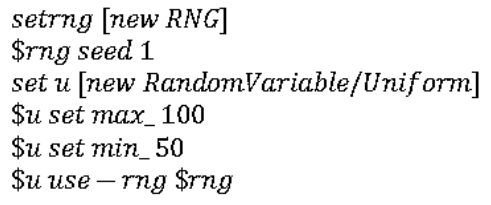

The "seed" value in the previous script, allows generating variable environments with different initial energies for nodes. At next, in order to generate uniform random initial energy for nodes, the following source code was added to the TCL script responsible for generating scenarios within NS2. This script will create a random number "u" varying between 50 and 100.After that, the random variable "u" will be associated with the initial energy of a node by the following TCL script:

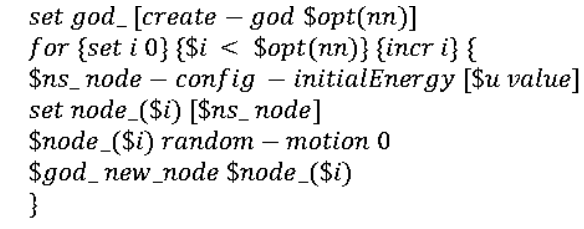

The implementation of these two scripts will allow us generating random scenarios with different residual energies distributions. For each "seed" value we can create a new energy distribution of nodes and we will test the LE-OLSR performances according to this distribution.

The Simulations were conducted using NS 2.35 [20, 21], under different mobility and density scenarios, where we varied node velocity and node density. Nodes in the simulation move according to Random Waypoint Mobility model each node is then assigned a particular trajectory, and the "two ray ground propagation model" is used [22]. We select the number of network nodes to be 60 in an area of $870 * 870$ square meters while changing the speed. We set the speed of nodes movement at $1 \mathrm{~m} / \mathrm{s}$ when varying the nodes number. The simulation duration for every scenario is 300 seconds. During each simulation, constant bit-rates (CBR) connections are generated, and each CBR connection produces 2 packets per second with a packet size of 512 bytes. Simulation parameters are summarized in Table 1.

As discussed, Table 1 illustrates that for the dynamic environment, three different energy scenarios will be simulated, depending on the "seed" value.

Table 1. Simulation parameters

\begin{tabular}{|c|c|c|c|c|}
\hline \multirow[b]{2}{*}{ Parameters } & \multicolumn{2}{|c|}{ Static parameters } & \multicolumn{2}{|c|}{ Dynamic parameters } \\
\hline & Number of nodes & Node speed & Number of nodes & Node speed \\
\hline Transmission Area & & & $870 \mathrm{~m} \times 870 \mathrm{~m}$ & \\
\hline Nodes Number & $20,60,80,100$ & 60 & $20,60,80,100$ & 60 \\
\hline Node Speed & $1 \mathrm{~m} / \mathrm{s}$ & $1,3,5,8 \mathrm{~m} / \mathrm{s}$ & $1 \mathrm{~m} / \mathrm{s}$ & $1,3,5,8 \mathrm{~m} / \mathrm{s}$ \\
\hline Simulation Time & & & $300 \mathrm{~s}$ & \\
\hline Traffic Type & & & CBR & \\
\hline Connections Number & & & 8 & \\
\hline Packet Rate & \multirow{8}{*}{\multicolumn{2}{|c|}{100 joules }} & 2 packets & \\
\hline Initial Energy & & & [50-100] joules, depend & NG ( seed1, seed3, seed5) \\
\hline Rx Power & & & $1.1 \mathrm{w}$ & \\
\hline Tx Power & & & $1.65 \mathrm{w}$ & \\
\hline Idle Power & & & $0.3 \mathrm{w}$ & \\
\hline Sleep Power & & & $0.042 \mathrm{w}$ & \\
\hline Routing Protocol & & & LE-OLSR, OLSR & \\
\hline Mac Layer & & & 802.11 & \\
\hline
\end{tabular}

\section{RESULTS AND DISCUSSION}

In what follows, we will study the behavior LE-OLSR and its performances in terms of QoS [23] of the mobile ad hoc networks, especially for variable environments. So, metrics chosen are:

a) Average delay: specifies the mean time taken for a packet to reach its destination. If the packet exceeds a certain delay time, it is considered as an undesired packet and it is automatically discarded by routers [24].

b) Control Overhead: The control overhead parameter determines the number of routing packets (Hello and TC) needed to be exchanged in order to deliver the payload (data packets) [25]. This metric is crucial in order to evaluate the performance of a routing protocol. An increase of control packets means 
more energy wasted by nodes and directly affects the network lifetime.

c) Dead Nodes: As discussed earlier, the willingness of a node measures its ability to forward traffic from a node to its neighbors [26]. Thus, it is a critical parameter to perform the MPRset of a node. A dead node has willingness in the interval of WILL_NEVER, so it can only send its data rather than routing traffic.

The section below depicts results gained by various simulations performed and the discussion area.

\subsection{Statistical analysis based on the average delay}

To corroborate our approach, we performed a statistical analysis based on calculating the correlation coefficient between LE-OLSR and OLSR, taking as variable the average delay for both; density and mobility. The average delay obtained by simulation is illustrated by Table 2. Table 3 below depicts the correlation coefficient, calculated for each case:

Table 2. Average delay in LE-OLSR vs OLSR

\begin{tabular}{ccccc}
\hline Density (nodes number) & 20 & 60 & 80 & 100 \\
\hline LE-OLSR & $1,16 \mathrm{E}-04$ & $6,57 \mathrm{E}-04$ & $9,31 \mathrm{E}-04$ & $1,26 \mathrm{E}-03$ \\
OLSR & $7,76 \mathrm{E}-05$ & $2,34 \mathrm{E}-03$ & $7,25 \mathrm{E}-03$ & $1,79 \mathrm{E}-02$ \\
Mobility (nodes speed) & 1 & 3 & 5 & 8 \\
LE-OLSR & $1,06 \mathrm{E}-04$ & $1,99 \mathrm{E}-04$ & $1,55 \mathrm{E}-04$ & $1,16 \mathrm{E}-04$ \\
OLSR & $2,92 \mathrm{E}-03$ & $3,15 \mathrm{E}-03$ & $1,50 \mathrm{E}-03$ & $2,09 \mathrm{E}-03$ \\
\hline
\end{tabular}

Table 3. Correlation coefficient for LE-OLSR and OSLR

\begin{tabular}{ccc}
\hline Environment & Protocol & Correlation coefficient \\
\hline Density & LE-OLSR & 0,995322592 \\
& OLSR & 0,789748591 \\
Mobility & LE-OLSR & 0,767007559 \\
& OLSR & 0,659930956 \\
\hline
\end{tabular}

Then we computed the correlation as follows [27]:

$$
\begin{aligned}
& r=\frac{n \sum x y-\sum x \sum y}{\sqrt{n \sum x^{2}-\left(\sum x\right)^{2}} \cdot \sqrt{n \sum y^{2}-\left(\sum y\right)^{2}}} \\
& r: \text { correlation coefficient } \\
& x: \text { nodesnumber }(20,40,60,80) \\
& y: \text { the average delay value } \\
& n: \text { number of samples (4 in our case) }
\end{aligned}
$$

Therefore, we can evaluate the strength of the relationship between the average delay and both density and mobility, in both two cases: OLSR and LE-OLSR. Figures 2 and 3 illustrate a comparison in terms of correlation between LE-OLSR and OLSR.

A correlation coefficient closer to 1 signifies that the relationship between the average delay and the density (mobility) is stronger. According to Table 3 and Figures 2 and 3, the obtained results show obviously that the variation of the packet delay in case of LE-OLSR is highly dependent on the density or the mobility of the network, compared to the basic OLSR protocol.

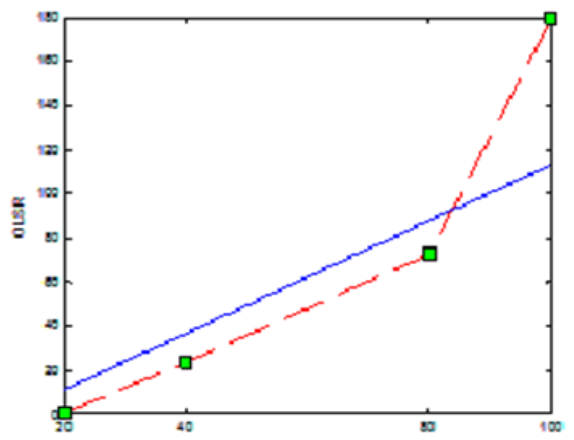

(a)

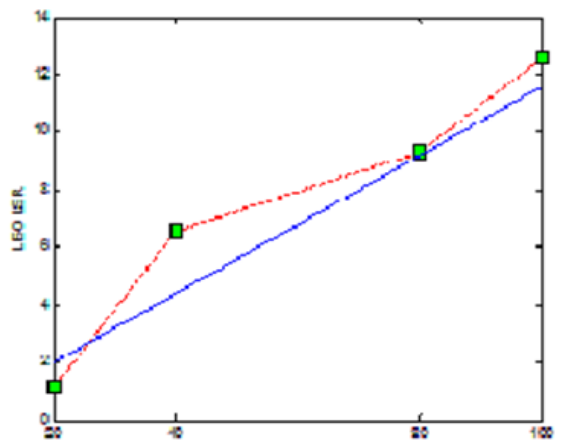

(b)

Figure 2. Linear regression plot of OLSR and LE-OLSR for Average delay Vs density (a) OLSR (b) LE-OLSR 


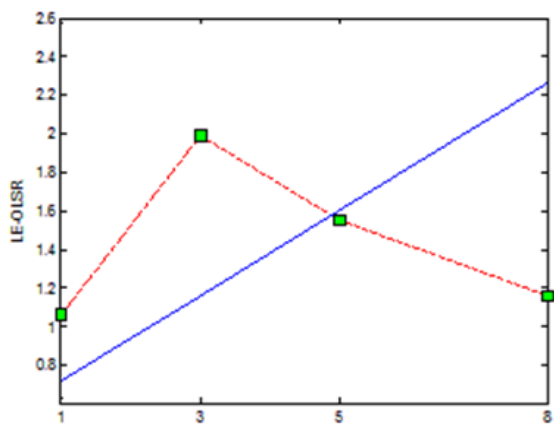

(a)

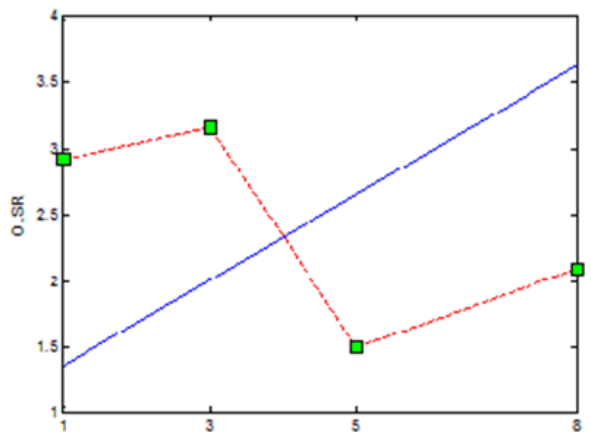

(b)

Figure 3. Linear regression plot of OLSR and LE-OLSR for Average delay Vs Mobility (a) OLSR (b) LE-OLSR

In case of density, the correlation coefficient " $r$ " is about 0,99for LE-OLSR instead of 0,78 for OLSR. This means that the behavior of LE-OLSR is linear and predictable when the network density increases, which is not the case for OLSR. When it comes to mobility, the correlation is less than the density for LE-OLSR but always much better than OLSR. These obtained results confirm that our approach LEOLSR is validated and outperforms the basic OLSR protocol, even for different energy constraints scenarios.

\subsection{QoS metric performances evaluation for LE-OLSR within different energy scenarios}

The Quality of Service (QoS) [27] is defined as a set of service requirements that needs to be guaranteed by the network while transporting a packet stream from a source to its destination. The network is expected to assure a set of measurable service attributes to users in terms of end-to-end performance, Power consumption and end to end delay which are more specific to mobile ad hoc networks [28, 29]. Furthermore, our concern is to study the efficiency of LE-OLSR in terms of energy consumption and the network lifetime.

As explained earlier, we performed scripts within NS2 to generate various scenarios, allowing us to study the behavior of LE-OLSR within realistic ad-hoc and sensor networks. Thus, we created three different random environments named (seed1, Seed3, seed5) and we compared the evolution of the QoS criterions when implementing LE-OLSR in both ideal and real environment.

\subsection{Effect of node density and node mobility on the control overhead}

Figure 4 illustrates the evolution of the control overhead when varying the initial residual energy of nodes for different scenarios. We notice that, the control overhead of the different scenarios increases when the nodes number becomes important and when the network seems highly mobile. This is due to the proactive scheme of OLSR, it can be explained by the fact that nodes exchange a huge number of control packets to build or maintain routes within the whole network to avoid link breakage when both density and mobility increase.

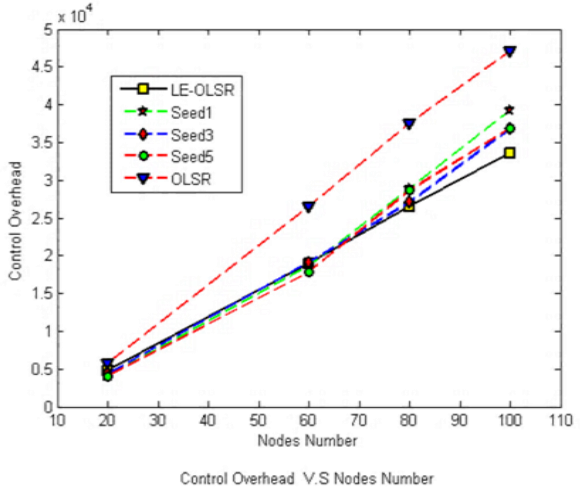

(a)

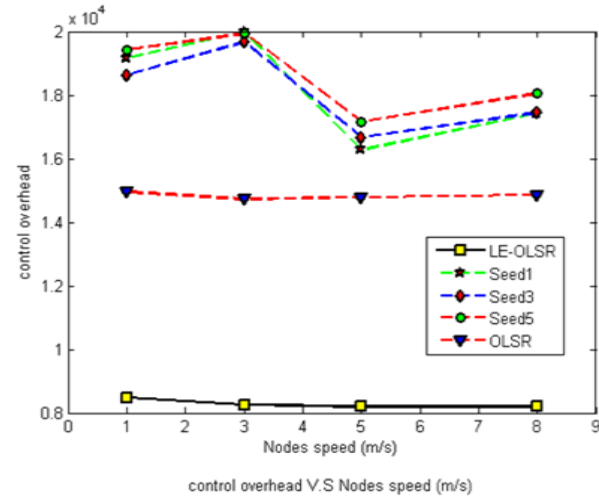

(b)

Figure 4. Effect of node density and node mobility on control overhead (a) network density (b) network mobility 
It's obvious according to the figure 4, that the control overhead is affected by the dynamic environment in both "density" and "mobility" case. In the static LE-OLSR the control overhead varies within the interval [4866, 33645]. However, it increases when implementing LE-OLSR within a random scenario where the interval is [4066,36866]. A lifetime efficient protocol should generate less control packets for transmitting the same amount of data packets. The rise of the control packets between the LE-OLSR and the three variable scenarios is about $11 \%$ for the density, and more than $30 \%$ in the mobility case. When density increases, LE-OLSR keeps its performances for both random and ideal ad hoc network. In case of mobility, we should take more care about the initial residual energy of nodes.

\subsection{Effect of node density and node mobility on dead nodes}

Dead nodes are nodes with a discharged battery (less than $5 \%$ of the initial residual energy in our case) due to their activity within the ad hoc network [30]. A dead node is not able to forward packets to its neighborhood. This means that an increase number of dead nodes, especially those which have a strategic position, generates more route failures and requires more calculation of route discovery, which produces additional control traffic that consumes more bandwidth and leaves less capacity for data traffic.

Figure 5, shows the variation of dead nodes for LE-OLSR in the ideal case and when generating random scenarios, for both density and mobility. According to the figure 5, the ratio of dead nodes is relatively stable for LE-OLSR, in the ideal case and when generating random scenarios. The dead node interval is between 11 and 68. Instead, for OLSR the dead nodes number increases drastically to reach 91. However, in the mobility case, dead nodes number for LE-OLSR in random scenarios exceeds the number of dead nodes for basic OLSR.

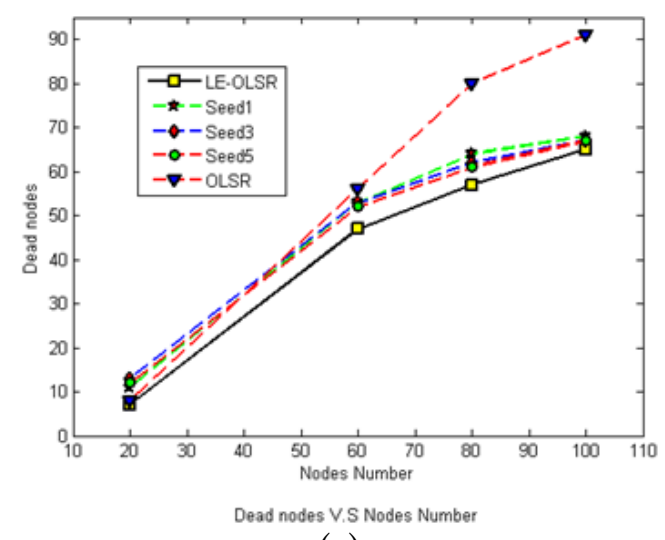

(a)

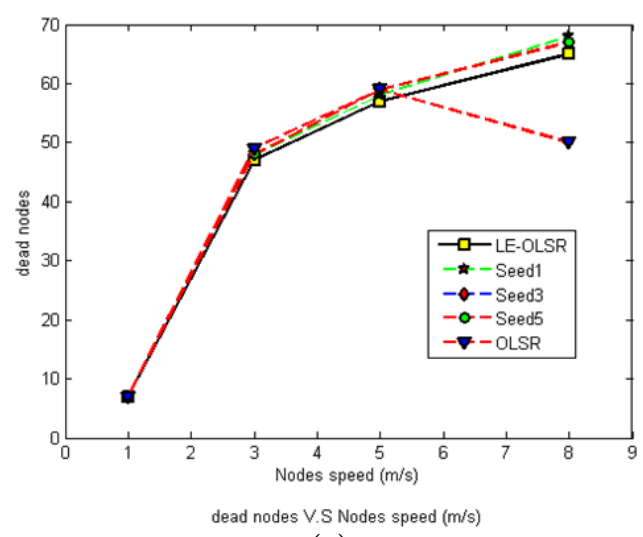

(b)

Figure 5. Effect of node density and node mobility on dead nodes (a) network density (b) network mobility

Identically to the evolution of the control overhead, we conclude that the LE-OLSR outperforms OLSR for both static and random scenarios when network becomes dense. However, we should pay attention to the initial energy constraints when implementing LE-OLSR in a highly mobile network.

\section{CONCLUSION}

The main objective of this paper was to study the behavior of LE-OLSR protocol in wireless ad hoc and sensor networks in different scenarios similar to the reality, where battery capacity level of nodes varies from one node to another.

In this work, we evaluated LE-OLSR protocol according to different QoS metrics; average delay, control overhead and dead nodes. The various performed simulations illustrate that LE-OLSR shows a slight decrease in its performance when we implement it in a realistic mobile ad hoc and wireless sensor environment.

In the density case, LE-OLSR kept its performance for both ideal and realistic environments and outperforms the basic OLSR. On the other hand, the mobility of nodes affects the LE-OLSR performances and leads to a degradation in the QoS. Based on the tolerance degree, we can say that LE-OLSR acts better in deterministic networks, where the initial residual energy of nodes is well known. 
The obtained results make LE-OLSR a strong candidate and very useful option for all type of a dense mobile wireless networks that implement a large number of mobile nodes. Our contribution may improve performance the existing networks, through our reliable concept of lifetime enhancement.

\section{REFERENCES}

[1] S. Jabbar,et al, "Analysis of Factors Affecting Energy Aware Routing in Wireless Sensor Network," Wireless Communications and Mobile Computing, vol. 2018, February 2018.

[2] S. Haoet al, "A Stable and Energy-Efficient Routing Algorithm Based on Learning Automata Theory for MANET," Journal of Communications and Information Networks, 2018.

[3] A. A. Alghamdi, et al, "Energy-efficient adaptive forwarding scheme for MANETs," IEEE Wireless days, 2016.

[4] H. Alani, et al, "Dynamic routing discovery scheme for high mobility in mobile ad hoc wireless networks," International Journal of Electrical and Computer Engineering (IJECE), vol. 10, no. 4, pp. 3702-3714, 2020.

[5] L.Fratta, et al, "Emerging trends and applications in ad hoc networks," Annals of Telecommunication vol. 73, pp. 547-548. 2018.

[6] A. Banerjee, et al, "Expected residual lifetime based ad hoc on-demand multipath routing protocol (ERL-AOMDV) in mobile ad hoc networks," International Journal of Information Technology, vol. 11, no. 4, pp. 727-733. 2018.

[7] B. Abidi, et al, "Security in wireless sensor networks," International Journal of Informatics and Communication Technology (IJ-ICT), vol. 8, no.1, pp. 13-18, 2019.

[8] D. K. Sharma, et al, "An Update based Energy-Efficient Reactive Routing Protocol for Mobile Ad Hoc Networks," International Journal in Computer Network and Information Security, vol. 11, pp. 17-26, 2013.

[9] DS. Sisodia, et al, "A Performance Review of Intra and Inter-Group MANET Routing Protocols under Varying Speed of Nodes," International Journal of Electrical and Computer Engineering (IJECE), vol. 7, no. 5, pp. 2721-2730, 2017.

[10] T. Clausen, et al, "Optimized Link State Routing Protocol (OLSR)," IETF RFC 3626, 2003.

[11] T. Clausen, et al, "Generalized Mobile Ad Hoc Network (MANET) Packet/Message Format," IETF RFC 5444. February 2009.

[12] T. Clausen, et al, "Representing Multi-Value Time in Mobile Ad Hoc Networks (MANETs)," IETF RFC 4597. March 2009.

[13] O.Barki, et al, "Improving the selection of MPRs in OLSR protocol: a survey of methods and techniques," International Journal of Electrical and Computer Engineering (IJECE), vol. 10, no. 1, pp. 288-295, 2020.

[14] M.Belkheir, et al, "Ad hoc Network Lifetime Enhancement by Energy Optimization," Ad Hoc \& Sensor Wireless Networks, vol. 28, no. 1-2, pp. 83-95, 2015.

[15] SAH. Belkhira, et al, "WRE-OLSR, a new scheme for enhancing the lifetime within ad hoc and wireless sensor networks," International journal of communication systems (IJCS), vol. 32, no. 11, 2019.

[16] A.Sahnoun, et al, "A Coalition-Formation Game Model for Energy-Efficient Routing in Mobile Ad-hoc Network," International Journal of Electrical and Computer Engineering (IJECE), vol. 8, no. 1, pp. 26-33, 2018,

[17] F. Lakrami, et al, "Energy and mobility in OLSR routing protocol," Journal of Selected Areas in Telecommunications (JSAT), March Edition, 2012.

[18] J.Dykes, et al, "Scripting Dynamic Maps: Some Examples and Experiences with Tcl/Tk," (eds) Multimedia Cartography. Springer, Berlin, 2019.

[19] RB.Aland, et al, "Process automation of simulation using Toolkit/ToolCommand Language (TK/TCL)scripting," 2nd International conference on Advances in Mechanical Engineering(ICAME), 2018.

[20] The Network Simulator (NS-2), http://www.isi.edu/nsnam/ns (official site of NS)

[21] N.Singh, et al, "Network Simulator NS2-2.35," International Journal of Advanced Research in Computer Science and Software Engineering, vol. 2, no. 5, May 2012.

[22] Alamsyah, et al, "MPR selection to the OLSR quality of service in MANET using minmax algorithm," International Journal of Electrical and Computer Engineering (IJECE), Vol. 9, No. 1, pp. 417-425, February 2019

[23] A.Lakas, et al, "A Multiconstrained QoS-Compliant Routing Scheme forHighway-Based Vehicular Networks," Wireless Communications and Mobile Computing, Volume 2019, Article ID 4521859, February 2019.

[24] SA.AlQhatani, et al, "Delay-Aware Resource Allocation for M2M Communications Over LTE-A Network," Arab J Sci Eng, vol. 44, pp. 3639-3653, 2019.

[25] MS.Shamaee, et al, "A smart proactive routing protocol in cognitive radio networks," International Journal of Ad Hoc and Ubiquitous Computing, vol. 31, no.2, pp. 83-102, 2019.

[26] H. Liang, et al, "Research on routing optimization of WSNs based on improved LEACH protocol," Journal of Wireless Communications Networks, 2019.

[27] M. Lin, et al, "TOPSIS Method Based on Correlation Coefficient and Entropy Measure for Linguistic Pythagorean Fuzzy Sets and Its Application to Multiple Attribute Decision Making," Complexity, vol. 2019, 2019.

[28] P. Sra and S.Chand, "QoS in Mobile Ad-Hoc Networks," Wireless Personal Communications, vol 105, pp.1599-1616, 2019.

[29] H. Elwahsh, et al, "A Novel Approach for Classifying MANETs Attacks with aNeutrosophic Intelligent System based on Genetic Algorithm," Security and Communication Networks, vol. 2018, 2018.

[30] M. Belkheir, et al, "An Energy Optimization Algorithm for Mobile Ad Hoc Network," International Journal of Soft Computing and Software Engineering (JSCSE), vol. 2, no. 10, pp. 22-26, 2012. 